\title{
Estimação de parâmetros genéticos e predição de valor genético aditivo de trigo utilizando modelos mistos
}

\author{
Adérico Júnior Badaró Pimentel(1), João Filipi Rodrigues Guimarães(1), Moacil Alves de Souza(1), \\ Marcos Deon Vilela de Resende ${ }^{(2)}$, Lisandra Magna Moura ${ }^{(1)}$, \\ João Romero do Amaral Santos de Carvalho Rocha ${ }^{(1)}$ e Guilherme Ribeiro ${ }^{(3)}$
}

\begin{abstract}
(1)Universidade Federal de Viçosa, Avenida Peter Henry Rolfs, s/no, CEP 36570-000 Viçosa, MG, Brasil. E-mail: adericojr@yahoo.com.br, filipiguimaraes@yahoo.com.br, moacil@ufv.br, lisandrammoura@yahoo.com.br, joao.rocha@ufv.br (2)Embrapa Florestas, Estrada da Ribeira, Km 111, CEP 83411-000 Colombo, PR, Brasil. E-mail: marcos.deon@gmail.com (3)Universidade Federal do Pampa, Campus Itaqui, Rua Luiz Joaquim de Sá Britto, s/nº, CEP 97650-000 Itaqui, RS, Brasil. E-mail: guilhermeribeiro@unipampa.edu.br
\end{abstract}

Resumo - O objetivo deste trabalho foi estimar os parâmetros genéticos e predizer o valor genético de populações e indivíduos oriundos de populações segregantes de trigo, com o uso da metodologia de modelos mistos ("restricted maximum likelihood"/"best linear unbiased prediction", REML/BLUP). Trinta e seis populações segregantes de trigo e quatro controles foram avaliados na geração $\mathrm{F}_{3}$, em delineamento de blocos ao acaso, com informações de indivíduo retiradas de dentro das parcelas. Os caracteres avaliados foram: produção de grãos, índice de colheita, número de perfilhos e altura de planta. Observou-se a existência de variabilidade genética entre populações em todos os caracteres avaliados. A herdabilidade média variou de 39,15 a 92,78\%, e a acurácia, de 62,57 a 96,32\%, na seleção de populações. A herdabilidade individual no sentido restrito foi baixa dentro das populações, em todos os caracteres. A acurácia na seleção individual apresentou magnitude média, quanto ao caráter altura de plantas, e baixa quanto aos demais caracteres. A herdabilidade individual contribui para maior ganho nos caracteres altura de planta e índice de colheita com o uso do BLUP individual, em comparação ao BLUP de populações. As populações segregantes Embrapa22/BRS207, Embrapa22/ VI98053, Embrapa22/IVI01041, BRS254/BRS207, BRS254/VI98053, BRS254/UFVT1Pioneiro e BRS264/ BRS207 destacam-se por apresentar valor genético aditivo elevado em dois ou mais caracteres.

Termos para indexação: Triticum aestivum, análise de deviance, dados desbalanceados, estratégias de melhoramento, população segregante, REML/BLUP.

\section{Estimation of genetic parameters and prediction of additive genetic value for wheat by mixed models}

\begin{abstract}
The objective of this work was to estimate the genetic parameters and to predict the genotypic value of populations and individuals from wheat segregating populations, using the methodology of mixed models (restricted maximum likelihood/best linear unbiased prediction, REML/BLUP). Thirty-six wheat segregating populations and four controls were evaluated in the $\mathrm{F}_{3}$ generation, in a randomized complete block design, with individual information taken from within the plots. The evaluated traits were: grain yield, harvest index, number of tillers, and plant height. Genetic variability between populations was observed for all evaluated traits. The mean heritability varied from 39.15 to $92.78 \%$, and accuracy varied from 62.57 to $96.32 \%$ in the selection of populations. The narrow-sense individual heritability was low within populations for all traits. The accuracy for individual selection had a moderate value for plant height, and low values for the other traits. Individual heritability contributes to a greater gain for the traits plant height and harvest index with the use of individual BLUP, in comparison to population BLUP. The segregating populations Embrapa22/BRS207, Embrapa22/VI98053, Embrapa22/IVI01041, BRS254/BRS207, BRS254/VI98053, BRS254/UFVT1Pioneiro, and BRS264/BRS207 stand out with high additive genetic value, for two or more traits.
\end{abstract}

Index terms: Triticum aestivum, deviance analysis, unbalanced data, breeding strategies, segregating populations, REML/BLUP.

\section{Introdução}

O melhoramento genético da cultura do trigo (Triticum aestivum L.) tem sido fundamental para o cultivo da espécie no país, em razão da disponibilização de cultivares com alto potencial produtivo, ampla adaptação e elevada aceitação pelo mercado consumidor. No Sul do Brasil, Nedel (1994)

Pesq. agropec. bras., Brasília, v.49, n.11, p.882-890, nov. 2014

DOI: 10.1590/S0100-204X2014001100007 
observou incremento da produtividade de grãos de $17,3 \mathrm{~kg} \mathrm{ha}^{-1}$ por ano, em cultivares lançadas entre 1940 e 1992. Em Minas Gerais, o ganho genético médio na produtividade de grãos, entre os anos de 1976 e 2005, foi de $48 \mathrm{~kg} \mathrm{ha}^{-1}$ por ano de trigo irrigado (Cargnin et al., 2008) e $37 \mathrm{~kg} \mathrm{ha}^{-1}$ por ano de trigo de sequeiro (Cargnin et al., 2009).

Apesar do expressivo êxito dos programas de melhoramento de trigo, quanto à avaliação, seleção e recomendação de novas cultivares, a superação dos níveis atuais de produtividade apresentados não é tarefa fácil para os melhoristas, principalmente por se tratar de um caráter de herança quantitativa. Assim, a busca de metodologias mais eficientes de seleção é a alternativa de efeito mais rápido, sobretudo quanto à produção de grãos, para a qual a variabilidade genética ainda está longe de ser exaurida (Cargnin et al., 2008, 2009). O uso de procedimentos genético-estatísticos mais refinados, como a metodologia de modelos mistos, é uma tendência no melhoramento genético de plantas. Estes procedimentos fornecem parâmetros adicionais relevantes para a identificação de genótipos superiores (Maia et al., 2011; Ramalho \& Araújo, 2011).

Um dos entraves à obtenção de ganhos genéticos mais expressivos no melhoramento de plantas é a baixa acurácia seletiva. Para resolver isto, o procedimento mais preciso de predição de valores genéticos é o BLUP (melhor predição linear não viesada), com uso de componentes de variância estimados via REML (máxima verossimilhança restrita) (Resende, 2004). O uso de modelos mistos do tipo REML/ BLUP é fundamental para a predição de valores genéticos aditivos e genotípicos, tanto intra quanto interpopulacionalmente (Resende, 2000). Mesmo em condições de experimentos desbalanceados, essa abordagem permite a predição acurada e não viesada dos valores genéticos, propicia informações específicas sobre o mérito da progênie, de indivíduos nas progênies e considera até mesmo o ambiente da repetição onde está localizado um indivíduo (Resende, 2007a). Assim, as propriedades do BLUP permitem maximização da acurácia seletiva, minimização do erro de predição, predição não viciada de valores genéticos, maximização do ganho genético - por ciclo de seleção - e maximização da probabilidade de seleção do melhor entre dois ou vários genótipos (Resende, 2004).

A eficiência do uso de modelos mistos no melhoramento de plantas anuais tem sido comprovada em diferentes culturas, como arroz (Borges et al., 2010), soja (Pinheiro et al., 2013), milho-pipoca (Freitas et al., 2013), feijão-caupi (Barros et al., 2011) e feijão-comum (Bertoldo et al., 2009).

Ramalho \& Araújo (2011) apontam a utilização de modelos mistos como estratégia adequada para maior eficiência do melhoramento de plantas autógamas, pela identificação de progênies ou linhas com maior mérito genotípico. Segundo Nunes et al. (2008) e Ramalho et al. (2013), o ganho genético com a seleção de plantas autógamas pode ser maximizado, quando se associa o método genealógico com o uso de modelos mistos. No melhoramento de Phaseolus vulgaris, Mendes et al. (2011) utilizaram predições realizadas via BLUP, para identificar progênies e indivíduos superiores dentro de populações segregantes. Chiorato et al. (2008) recomendam o REML/BLUP para orientação de programas de melhoramento de feijão, e Coimbra et al. (2005), para o melhoramento de aveia.

Em algumas espécies autógamas, as avaliações de plantas individuais têm sido realizadas em populações $\mathrm{F}_{3}$, consideradas adequadas para seleção, pelo fato de que $75 \%\left(1,5 \sigma_{a}^{2}\right)$ da variação aditiva total $\left(2 \sigma_{a}^{2}\right)$ disponível em $\mathrm{F}_{\infty}$ já se encontra disponível em $\mathrm{F}_{3}$ (Resende, 2002). Para a seleção neste caso, o BLUP pode ser derivado do delineamento de blocos ao acaso, com várias plantas por parcela.

O uso da metodologia REML/BLUP, na rotina dos programas de melhoramento de trigo, ainda é pouco frequente, e é igualmente incomum a estimação de componentes de variância e efeitos genéticos aditivos (Oakey et al., 2006).

O objetivo deste trabalho foi estimar os parâmetros genéticos e predizer o valor genético de populações e indivíduos oriundos de populações segregantes de trigo, com o uso da metodologia de modelos mistos ("restricted maximum likelihood"/"best linear unbiased prediction", REML/BLUP).

\section{Material e Métodos}

O experimento foi realizado no campo experimental Professor Diogo Alves de Mello, Universidade Federal de Viçosa, em Viçosa, MG (2045'14" S; 4252'55" W, a $648 \mathrm{~m}$ de altitude), entre maio e setembro de 2009.

Foram avaliadas 36 populações segregantes da geração $F_{3}$, juntamente com as cultivares 'MGS 1 Aliança', 'Embrapa 22', 'UFVT 1 Pioneiro' e 'BRS 264',

Pesq. agropec. bras., Brasília, v.49, n.11, p.882-890, nov. 2014 DOI: 10.1590/S0100-204X2014001100007 
utilizadas como testemunhas, para estimar os efeitos de ambiente (Tabela 1). Os tratamentos genéticos (populações e testemunhas) foram dispostos em delineamento de blocos ao acaso, com informações de indivíduo tiradas de dentro da parcela, e o uso de três

Tabela 1. Relação das populações segregantes de trigo, com seus respectivos cruzamentos e número de plantas, avaliadas quanto aos caracteres produtividade de grãos $(\mathrm{PG})$, índice de colheita (IC), número de perfilhos (NP) e altura de planta (AP).

\begin{tabular}{|c|c|c|c|c|c|}
\hline \multirow[t]{2}{*}{ População } & \multirow[t]{2}{*}{ Cruzamento } & \multicolumn{4}{|c|}{ Número de plantas } \\
\hline & & PG & $\mathrm{IC}$ & $\mathrm{NP}$ & AP \\
\hline 1 & Embrapa22/Anahuac 75 & 225 & 225 & 233 & 233 \\
\hline 2 & Embrapa22/MGS 1 Aliança & 232 & 232 & 237 & 237 \\
\hline 3 & Embrapa22/BRS207 & 214 & 214 & 216 & 216 \\
\hline 4 & Embrapa22/VI98053 & 243 & 243 & 243 & 243 \\
\hline 5 & Embrapa22/IVI01041 & 232 & 232 & 233 & 233 \\
\hline 6 & Embrapa22/UFVT 1 Pioneiro & 258 & 258 & 259 & 259 \\
\hline 7 & Embrapa42/Anahuac 75 & 240 & 240 & 247 & 247 \\
\hline 8 & Embrapa42/MGS 1 Aliança & 247 & 247 & 255 & 255 \\
\hline 9 & Embrapa42/BRS207 & 223 & 223 & 225 & 225 \\
\hline 10 & Embrapa42/VI98053 & 220 & 220 & 224 & 224 \\
\hline 11 & Embrapa42/IVI01041 & 219 & 219 & 232 & 232 \\
\hline 12 & Embrapa42/UFVT 1 Pioneiro & 241 & 241 & 246 & 246 \\
\hline 13 & BRS254/Anahuac 75 & 225 & 225 & 226 & 226 \\
\hline 14 & BRS254/MGS 1 Aliança & 253 & 253 & 253 & 253 \\
\hline 15 & BRS254/BRS207 & 228 & 228 & 228 & 228 \\
\hline 16 & BRS254/VI98053 & 241 & 241 & 243 & 243 \\
\hline 17 & BRS254/IVI01041 & 215 & 215 & 216 & 216 \\
\hline 18 & BRS254/UFVT 1 Pioneiro & 241 & 241 & 244 & 244 \\
\hline 19 & BRS264/Anahuac 75 & 236 & 236 & 239 & 239 \\
\hline 20 & BRS264/MGS 1 Aliança & 260 & 260 & 263 & 263 \\
\hline 21 & BRS264/BRS207 & 223 & 223 & 226 & 226 \\
\hline 22 & BRS264/VI98053 & 250 & 250 & 252 & 252 \\
\hline 23 & BRS264/IVI01041 & 209 & 209 & 212 & 212 \\
\hline 24 & BRS264/UFVT 1 Pioneiro & 237 & 237 & 240 & 240 \\
\hline 25 & IAC24-Tucuruí/Anahuac 75 & 229 & 229 & 233 & 233 \\
\hline 26 & IAC24-Tucuruí/ MGS 1 Aliança & 258 & 258 & 260 & 260 \\
\hline 27 & IAC24-Tucuruí/BRS207 & 221 & 221 & 224 & 224 \\
\hline 28 & IAC24-Tucuruí/VI98053 & 240 & 240 & 244 & 244 \\
\hline 29 & IAC24-Tucuruí/IVI01041 & 206 & 206 & 219 & 219 \\
\hline 30 & IAC24-Tucuruí/ UFVT 1 Pioneiro & 239 & 239 & 240 & 240 \\
\hline 31 & IAC364-Tucuruí III/Anahuac 75 & 217 & 217 & 219 & 219 \\
\hline 32 & IAC364-Tucuruí III/MGS 1 Aliança & 232 & 232 & 236 & 236 \\
\hline 33 & IAC364-Tucuruí III/BRS207 & 223 & 223 & 224 & 224 \\
\hline 34 & IAC364-Tucuruí III/VI98053 & 250 & 250 & 251 & 251 \\
\hline 35 & IAC364-Tucuruí III/IVI01041 & 202 & 202 & 213 & 213 \\
\hline 36 & IAC364-Tucuruí III/UFVT 1 Pioneiro & 231 & 231 & 241 & 241 \\
\hline Test & Embrapa 22 & 246 & 246 & 248 & 248 \\
\hline Test & BRS 264 & 220 & 220 & 222 & 222 \\
\hline Test & MGS 1 Aliança & 252 & 252 & 252 & 252 \\
\hline Test & UFVT 1 Pioneiro & 213 & 213 & 212 & 212 \\
\hline Total & & 9.291 & 9.291 & 9.430 & 9.430 \\
\hline
\end{tabular}

Test, testemunha. repetições por tratamento. A parcela experimental foi composta por duas linhas de 5,0 m de comprimento, espaçadas em $0,20 \mathrm{~m}$, e a densidade de semeadura foi de 50 sementes aptas por metro quadrado, o que totalizou 100 plantas por parcela.

Foram realizadas observações fenotípicas, em cada planta, quanto aos seguintes caracteres: altura de planta $(\mathrm{cm})$, medida do coleto até o ápice da espiga, tendo-se excluído as aristas; índice de colheita, obtido pela razão entre a massa de matéria seca de grãos e da parte aérea; produtividade de grãos (g por planta), determinada após beneficiamento e correção da umidade para 13\%; e número de perfilhos por planta. Em razão de falhas no estande, o número de plantas avaliadas variou entre tratamentos e caracteres, conforme especificado na Tabela 1.

A adubação foi realizada de acordo com a análise de solo: $250 \mathrm{~kg} \mathrm{ha}^{-1}$ de N-P-K 08-28-16, aplicados ao sulco de semeadura; e $50 \mathrm{~kg} \mathrm{ha}^{-1}$ de $\mathrm{N}$, em cobertura, no início do perfilhamento. Os demais tratos culturais seguiram as recomendações técnicas para cultivo de trigo irrigado (Reunião da Comissão Brasileira de Pesquisa de Trigo e Triticale, 2008).

De posse das informações fenotípicas, o programa Selegen-REML/BLUP (Resende, 2007b) foi utilizado, para estimar os parâmetros e predizer os valores genéticos aditivos. $\mathrm{O}$ procedimento adotado no programa para a predição dos valores genéticos foi o BLUP, com uso das estimativas de variâncias obtidas pelo REML (Resende, 2007a).

Para estimar e predizer os parâmetros genéticos, foram consideradas as informações individuais dentro das parcelas. Na geração $\mathrm{F}_{3}, 66,6 \%$ da variabilidade encontra-se entre populações, e 33,3\% dentro das populações; ou seja, a variação entre populações contempla a variância genética aditiva uma vez, enquanto a variação dentro de populações a contempla meia vez (Resende, 2007a).

O modelo genético-estatístico do Selegen utilizado para estimar os componentes de variância e predizer os valores genotípicos foi o número 61 (Resende, 2007b), com a seguinte descrição: $\mathrm{y}=\mathrm{Xr}+\mathrm{Za}+\mathrm{Wp}+\mathrm{Tb}+\varepsilon$; em que y é o vetor de dados; $r$ é o vetor dos efeitos de populações e testemunhas, considerados como de efeitos fixos; a é o vetor dos efeitos genéticos aditivos individuais (assumidos como aleatórios); p é o vetor dos efeitos de parcela (assumidos como aleatórios); b é vetor dos efeitos de blocos (aleatórios); $\varepsilon$ é o 
vetor de erros ou resíduos (aleatórios); e X, Z, W, T são matrizes de incidência para os efeitos $r, a, p$ e b, respectivamente.

Na distribuição e estrutura de médias e variâncias, consideraram-se as seguintes condições:

$\mathrm{y}|\mathrm{r}, \mathrm{V} \sim \mathrm{N}(\mathrm{Xr}, \mathrm{V}) ; \mathrm{a}| \sigma_{\mathrm{a}}^{2} \sim \mathrm{N}\left(0, \mathrm{I} \sigma_{\mathrm{a}}^{2}\right) ; \mathrm{p} \mid \sigma_{\mathrm{p}}^{2} \sim \mathrm{N}\left(0, \mathrm{I} \sigma_{\mathrm{p}}^{2}\right) ;$

$\mathrm{b}\left|\sigma_{\mathrm{b}}^{2} \sim \mathrm{N}\left(0, \mathrm{I} \sigma_{\mathrm{b}}^{2}\right) ; \mathrm{e}\right| \sigma_{\mathrm{e}}^{2} \sim \mathrm{N}\left(0, \mathrm{I} \sigma_{\mathrm{e}}^{2}\right) ; \operatorname{Cov}=\left(\mathrm{a}, \mathrm{p}^{\prime}\right)=0$;

$\operatorname{Cov}=\left(a, b^{\prime}\right)=0 ; \operatorname{Cov}=\left(a, e^{\prime}\right)=0 ; \operatorname{Cov}=\left(p, b^{\prime}\right)=0$;

$\operatorname{Cov}=\left(p, e^{\prime}\right)=0 ;$ e $\operatorname{Cov}=\left(b, e^{\prime}\right)=0$;

ou seja:

$$
\mathrm{E}\left[\begin{array}{l}
\mathrm{y} \\
\mathrm{a} \\
\mathrm{p} \\
\mathrm{b} \\
\mathrm{e}
\end{array}\right]=\left[\begin{array}{l}
\mathrm{Xr} \\
0 \\
0 \\
0 \\
0
\end{array}\right] \text { e Var }\left[\begin{array}{l}
\mathrm{y} \\
\mathrm{a} \\
\mathrm{p} \\
\mathrm{b} \\
\mathrm{e}
\end{array}\right]=\left[\begin{array}{ccccc}
\mathrm{V} & \mathrm{ZG} & \mathrm{WP} & \mathrm{TB} & \mathrm{R} \\
\mathrm{GZ} & \mathrm{G} & 0 & 0 & 0 \\
\mathrm{PW} & 0 & \mathrm{P} & 0 & 0 \\
\mathrm{BT}^{\prime} & 0 & 0 & \mathrm{~B} & 0 \\
\mathrm{R} & 0 & 0 & 0 & \mathrm{R}
\end{array}\right]
$$

em que:

$$
\begin{aligned}
& \mathrm{G}=\mathrm{I} \sigma_{\mathrm{a}}^{2} ; \mathrm{R}=\mathrm{I} \sigma_{\mathrm{e}}^{2} ; \mathrm{P}=\mathrm{I} \sigma_{\mathrm{p}}^{2} ; \mathrm{B}=\mathrm{I} \sigma_{\mathrm{b}}^{2} ; \\
& \mathrm{V}=\mathrm{ZI} \sigma_{\mathrm{a}}^{2} \mathrm{Z}^{\prime}+\mathrm{WI} \sigma_{\mathrm{p}}^{2} \mathrm{~W}^{\prime}+\mathrm{TI} \sigma_{\mathrm{b}}^{2} \mathrm{~T}^{\prime}+\mathrm{I} \sigma_{\mathrm{e}}^{2}
\end{aligned}
$$

As equações de modelo misto para a predição BLUP dos valores genéticos individuais equivalem a:

$$
\left[\begin{array}{cccc}
X^{\prime} X & X^{\prime} Z & X^{\prime} W & X^{\prime} T \\
Z^{\prime} X & Z^{\prime} Z+I \lambda_{1} & Z^{\prime} W & Z^{\prime} T \\
W^{\prime} X & W^{\prime} Z & W^{\prime} W+I \lambda_{2} & W^{\prime} T \\
T^{\prime} X & T^{\prime} Z & T^{\prime} W & T^{\prime} T+I \lambda_{3}
\end{array}\right] \times\left[\begin{array}{c}
\hat{r} \\
\tilde{a} \\
\tilde{p} \\
\tilde{b}
\end{array}\right]=\left[\begin{array}{c}
X^{\prime} y \\
Z^{\prime} y \\
W^{\prime} y \\
T^{\prime} y
\end{array}\right]
$$

em que:

$\lambda_{1}=\frac{\sigma_{\mathrm{e}}^{2}}{\sigma_{\mathrm{a}}^{2}}=\frac{1-\mathrm{h}^{2}-\mathrm{C}_{\mathrm{p}}^{2}-\mathrm{C}_{\mathrm{b}}^{2}}{\mathrm{~h}^{2}} ; \lambda_{2}=\frac{\sigma_{\mathrm{e}}^{2}}{\sigma_{\mathrm{p}}^{2}}=\frac{1-\mathrm{h}^{2}-\mathrm{C}_{\mathrm{p}}^{2}-\mathrm{C}_{\mathrm{b}}^{2}}{\mathrm{C}_{\mathrm{p}}^{2}} ;$

$\lambda_{3}=\frac{\sigma_{\mathrm{e}}^{2}}{\sigma_{\mathrm{b}}^{2}}=\frac{1-\mathrm{h}^{2}-\mathrm{C}_{\mathrm{p}}^{2}-\mathrm{C}_{\mathrm{b}}^{2}}{\mathrm{C}_{\mathrm{b}}^{2}}$;

$\mathrm{h}^{2}=\sigma_{\mathrm{a}}^{2} / \sigma_{\mathrm{a}}^{2}+\sigma_{\mathrm{p}}^{2}+\sigma_{\mathrm{b}}^{2}+\sigma_{\mathrm{e}}^{2}$ é a herdabilidade individual no sentido restrito;

$\mathrm{C}_{\mathrm{p}}^{2}=\sigma_{\mathrm{p}}^{2} / \sigma_{\mathrm{a}}^{2}+\sigma_{\mathrm{p}}^{2}+\sigma_{\mathrm{b}}^{2}+\sigma_{\mathrm{e}}^{2}$ é a correlação, em razão do ambiente comum da parcela; $\mathrm{e}$

$C_{b}^{2}=\sigma_{b}^{2} / \sigma_{a}^{2}+\sigma_{p}^{2}+\sigma_{b}^{2}+\sigma_{\mathrm{e}}^{2}$ é a correlação, em razão do ambiente comum do bloco.

Os estimadores iterativos dos componentes de variância foram obtidos por REML, via algoritmo EM:

$\sigma_{\mathrm{e}}^{2}=\left[\mathrm{y}^{\prime} \mathrm{y}-\widehat{\mathrm{r}} \mathrm{X}^{\prime} \mathrm{y}-\tilde{\mathrm{a}} \mathrm{Z}^{\prime} \mathrm{y}-\tilde{\mathrm{p}} \mathrm{W} 1 \mathrm{y}-\tilde{\mathrm{b}} \mathrm{T}^{\prime} \mathrm{y}\right] /[\mathrm{N}-\mathrm{r}(\mathrm{x})]$;

$\sigma_{\mathrm{a}}^{2}=\left[\tilde{a}{ }^{\prime} \tilde{a}+\sigma_{\mathrm{e}}^{2} \operatorname{tr} C^{22}\right] / \mathrm{q}$; $\sigma_{\mathrm{p}}^{2}=\left[\tilde{\mathrm{p}}^{\prime} \tilde{\mathrm{p}}+\sigma_{\mathrm{e}}^{2} \operatorname{tr} \mathrm{C}^{33}\right] / \mathrm{s} ; \sigma_{\mathrm{b}}^{2}=\left[\tilde{\mathrm{b}} \tilde{\mathrm{b}}^{\tilde{b}}+\sigma_{\mathrm{e}}^{2} \operatorname{tr} \mathrm{C}^{44}\right] / \eta ;$

e $\mathrm{C}^{22}, \mathrm{C}^{33}$ e $\mathrm{C}^{44}$, advêm de:

$C^{-1}=\left[\begin{array}{llll}C^{11} & C^{12} & C^{13} & C^{14} \\ C^{21} & C^{22} & C^{23} & C^{24} \\ C^{31} & C^{32} & C^{33} & C^{34} \\ C^{41} & C^{42} & C^{43} & C^{44}\end{array}\right]=\left[\begin{array}{llll}C^{11} & C^{12} & C^{13} & C^{14} \\ C^{21} & C^{22} & C^{23} & C^{24} \\ C^{31} & C^{32} & C^{33} & C^{34} \\ C^{41} & C^{42} & C^{43} & C^{44}\end{array}\right]$

em que: C é matriz dos coeficientes das equações de modelo misto; tr é operador-traço matricial; $\mathrm{r}(\mathrm{x})$ é posto da matriz $\mathrm{X} ; \mathrm{N}, \mathrm{q}$, s e $\eta$ correspondem ao número total de dados, de indivíduos, de parcelas e de blocos, respectivamente.

Utilizando-se o índice multiefeitos, derivado por Resende \& Higa (1994), tem-se que o índice ótimo ou BLUP, neste caso, é dado por:

$$
\begin{aligned}
& I_{m}=b_{1} \delta_{i j k}+b_{2} g_{i}+b_{3} c_{i j}=b_{1}\left(Y_{i j k}-\bar{Y}_{i j .}\right) \\
& +b_{2}\left(\bar{Y}_{i . .}-\bar{Y}_{\ldots}\right)+b_{3}\left(\bar{Y}_{i j .}-\bar{Y}_{i . .}-\bar{Y}_{. j .}+\bar{Y}_{. .}\right)
\end{aligned}
$$

em que:

$\mathrm{b}_{1}=(1 / 2) \sigma_{\mathrm{a}}^{2} / \widehat{\sigma}_{\delta}^{2}$ é a herdabilidade do efeito de indivíduo dentro de parcela;

$$
\mathrm{b}_{2}=\frac{(2 \mathrm{nb}+1)}{\frac{2 \mathrm{nb}}{\sigma_{\mathrm{f}}^{2}+\sigma_{\mathrm{p}}^{2} / \mathrm{b}+\sigma_{\delta}^{2} / \mathrm{nb}}} \sigma_{\mathrm{a}}^{2}
$$

é a herdabilidade do efeito de populações; e

$$
\mathrm{b}_{3}=\frac{[(1 / 2) / \mathrm{n}] \sigma_{\mathrm{a}}^{2}}{\sigma_{\mathrm{c}}^{2}+\sigma_{\delta}^{2} / \mathrm{n}}
$$

é a herdabilidade do efeito de parcela.

Os componentes de variância $\sigma_{\mathrm{f}}^{2}, \sigma_{\mathrm{p}}^{2}$ e $\sigma_{\delta}^{2}$, referem-se às variâncias entre populações, entre parcelas e dentro de parcelas, respectivamente; enquanto as quantidades $\mathrm{n}$ e b referem-se aos números de indivíduos por parcela e número de blocos, respectivamente.

A estimação de $\sigma_{a}^{2}$ com dados apenas da geração $\mathrm{F}_{3}$ implica assumir $0,25 \sigma_{\mathrm{d}}^{2}$, com tendência a zero, na variação entre e dentro de progênies. Entretanto, mesmo sem esta suposição, a presença desta pequena fração da variância de dominância $\left(\sigma_{d}^{2}\right)$ não deverá afetar o ranking pelo BLUP, visto que as duas herdabilidades são usadas no cômputo de seus valores 
genéticos. Com as duas estimativas inflacionadas pela mesma quantidade, a proporcionalidade entre elas não será afetada (Resende, 2007b).

$\mathrm{Na}$ metodologia de modelos mistos com dados desbalanceados, os efeitos do modelo não devem ser testados via teste $F$, tal como se faz no método da análise de variância (Resende, 2007a). Neste caso, o teste recomendado para os efeitos aleatórios é o teste da razão de verossimilhança (LRT), por meio de uma análise de deviance. Esta análise, sugerida por Resende (2007a), representa uma generalização da análise de variância clássica, para casos balanceados e desbalanceados, e indica a qualidade do ajuste do modelo. A deviance é uma estatística derivada da razão entre as verossimilhanças do modelo completo, em relação ao modelo sem o efeito que se deseja testar.

\section{Resultados e Discussão}

A análise de deviance mostrou efeito significativo de genótipos quanto à produtividade de grãos $(\mathrm{p}<0,10)$, e quanto ao índice de colheita, ao número de perfilhos e à altura de plantas $(\mathrm{p}<0,01)$. Portanto, os respectivos componentes de variância aditiva e coeficientes de herdabilidade foram significativamente diferentes de zero, o que mostra a existência de variabilidade genética para esses caracteres. Esses resultados revelam a possibilidade de seleção, entre populações, de todos os caracteres.

Com exceção da produtividade de grãos, os demais caracteres apresentaram elevada herdabilidade de médias de população, que variou de 67,78 a $92,78 \%$. Esses valores possibilitam alta precisão na seleção de populações, com acurácia seletiva entre 82,33 e $96,32 \%$, de magnitude alta a muito alta, de acordo com Resende \& Duarte (2007). A produção de grãos apresentou herdabilidade de médias de população inferior a $40 \%$, e acurácia moderada.

A acurácia seletiva reflete a qualidade das informações e dos procedimentos utilizados na predição dos valores genéticos. Essa medida está associada à precisão da seleção e refere-se à correlação entre valores genéticos preditos e valores genéticos verdadeiros dos indivíduos. Quanto maior a acurácia seletiva na avaliação de um indivíduo, maior é a confiança na avaliação e no valor genético predito para o indivíduo. Portanto, devem-se destacar os resultados para índice de colheita e altura de plantas, que apresentaram valores expressivos de acurácia e de herdabilidade de médias, o que mostra elevada variabilidade genética aditiva, precisão na identificação e possibilidade de sucesso na seleção de populações.

$\mathrm{Na}$ seleção dentro de populações, a herdabilidade individual no sentido restrito - ou herdabilidade aditiva dentro de populações - apresentou valores baixos em todos os caracteres (Resende, 2002); na seleção massal, apresentou valores médios, para o caráter altura de planta, e baixos para os demais caracteres (Tabela 2). Esses baixos valores sugerem que não há condições favoráveis para a seleção de plantas individuais. Os valores de acurácia na seleção individual variaram de baixos a moderados, segundo a classificação de Resende \& Duarte (2007).

Herdabilidades individuais de baixa magnitude dentro de populações podem ser interpretadas como informação adicional às herdabilidades entre populações, quando o método BLUP individual é usado. Assim, as herdabilidades individuais dentro de populações quanto ao índice de colheita e altura de plantas apresentaram magnitude considerável - de 11,29 e 10,39\%, respectivamente (Tabela 2). Esses valores contribuíram para maior ganho com o BLUP individual, em comparação ao BLUP de populações.

A avaliação de indivíduos com o BLUP deu maior peso à informação baseada na média de populações. Todavia, houve contribuição da seleção dentro de populações, evidenciada pelo acréscimo nos valores de acurácia, quando são comparadas a acurácia na seleção de populações e a acurácia na seleção combinada entre e dentro de populações, com uso do BLUP individual. A eficiência do uso da informação de dentro das populações - obtida pela razão entre a acurácia na seleção combinada entre e dentro e a acurácia na seleção entre populações - foi de 1,01 quanto ao número de perfilhos, 1,03 ao índice de colheita, e 1,03 quanto à altura de plantas (Tabela 2). Nessas condições, a seleção pelo BLUP individual proporcionaria ganhos adicionais de $1 \%$ para o número de perfilhos e de $3 \%$ para o índice de colheita e a altura de plantas. Quanto à produtividade de grãos, a eficiência não se alterou, uma vez que a herdabilidade individual dentro de populações foi quase nula.

O coeficiente de variação genética aditiva individual (CVgi) quantifica a magnitude da variação genética disponível para seleção e, portanto, altos valores são desejáveis. A razão entre CVgi e CVe (coeficiente de variação experimental) permite obter o coeficiente de variação relativa $(\mathrm{CVr})$, cujo valor pode indicar 
uma situação favorável à seleção, se superior a 1,0 (Vencovsky \& Barriga, 1992), e propiciar inferências com alta acurácia e precisão (Resende \& Duarte, 2007). Para os caracteres índice de colheita e altura de plantas, o valor do coeficiente de variação relativo foi superior a 1,0, o que indicou grande possibilidade de sucesso com a seleção entre progênies, visto que esse parâmetro indica que a variação genética entre progênies é bem maior do que a variação ambiental. O contrário ocorreu com os caracteres produtividade de grãos e número de perfilhos (Tabela 2).

De acordo com Ramalho et al. (2013), o sucesso na seleção de progênies superiores está intrinsecamente ligado à experimentação adequada, pois, para a análise estatística resultar em boas predições de BLUP, é indispensável que haja boas estimativas dos componentes de variância.

O coeficiente de variação experimental (CVe) foi considerado baixo, o que mostra boa precisão do experimento (Tabela 2). O coeficiente de determinação dos efeitos de parcela (c2) também é uma medida da qualidade experimental e mede a variação ambiental entre parcelas dentro de blocos. Valores elevados de c2 significam alta variabilidade entre parcelas, dentro dos blocos, e alta correlação ambiental entre observações, dentro da parcela.
Os valores obtidos de c2 foram baixos e variaram de $1,39 \%$, quanto à altura de planta, a $8,29 \%$, quanto ao índice de colheita. Portanto, menos de $10 \%$ da variação fenotípica total ocorreu em razão da variação ambiental entre parcelas dentro dos blocos (Tabela 2). Assim, a variabilidade ambiental, entre parcelas dentro dos blocos e a correlação ambiental, entre observações dentro da parcela, foram baixas. A contribuição expressiva da variância fenotípica, observada dentro de parcela, para a variância fenotípica total, ratifica esse resultado e mostra que a maioria da variação ambiental encontra-se dentro de parcelas.

Quando se analisa o efeito de tratamentos, tomando-os como aleatórios, os testes de comparações múltiplas entre médias de tratamentos não devem ser utilizados, uma vez que estes testes são derivados de uma suposição de efeitos fixos de tratamentos e, também, porque produzem inferências sobre médias fenotípicas e não médias genotípicas (Resende, 2004). O que se obtém da análise de modelos mistos é um ordenamento decrescente dos genótipos, de acordo com seus valores genéticos (Duarte \& Vencovsky, 2001) corrigidos e penalizados, para a ocorrência de efeitos ambientais.

$\mathrm{Na}$ análise das dez melhores populações segregantes, cinco $(3,5,15,18$ e 21$)$ apresentaram

Tabela 2. Componentes de variância e parâmetros genéticos estimados para os caracteres produtividade de grãos (g por planta), índice de colheita, número de perfilhos e altura de planta $(\mathrm{cm})$, em populações segregantes de trigo.

\begin{tabular}{|c|c|c|c|c|}
\hline Parâmetro & $\begin{array}{l}\text { Produtividade } \\
\text { de grãos }\end{array}$ & $\begin{array}{c}\text { Índice de } \\
\text { colheita }\end{array}$ & $\begin{array}{c}\text { Número de } \\
\text { perfilhos }\end{array}$ & $\begin{array}{c}\text { Altura de } \\
\text { planta }\end{array}$ \\
\hline Variância genética aditiva entre populações $F_{3}$ & 0,2075 & 0,0007 & 0,1143 & 37,9764 \\
\hline Variância ambiental entre parcelas & 0,7772 & 0,0004 & 0,0992 & 3,1215 \\
\hline Variância entre blocos & 0,5075 & 0,0007 & 0,0008 & 0,2848 \\
\hline Variância fenotípica dentro da parcela & 11,1710 & 0,0031 & 3,8872 & 182,7484 \\
\hline Variância fenotípica total & 126,631 & 0,0049 & 4,1015 & 224,1312 \\
\hline Variância aditiva dentro de populações & 0,1038 & 0,0004 & 0,0572 & 18,9882 \\
\hline Herdabilidade individual no sentido restrito (massal, \%) & 1,64 & 13,55 & 2,79 & 16,94 \\
\hline Herdabilidade individual no sentido restrito dentro de populações (\%) & 0,00 & 11,29 & 1,47 & 10,39 \\
\hline Herdabilidade em nível de médias (\%) & 39,15 & 79,41 & 67,78 & 92,78 \\
\hline Acurácia na seleção individual (massal, \%) & 12,81 & 36,54 & 16,70 & 41,16 \\
\hline Acurácia na seleção de populações (\%) & 62,57 & 89,11 & 82,33 & 96,32 \\
\hline Acurácia na seleção combinada entre e dentro (BLUP, \%) & 62,57 & 92,22 & 82,78 & 98,98 \\
\hline Eficiência com seleção pelo BLUP entre e dentro de populações & 1,00 & 1,03 & 1,01 & 1,03 \\
\hline Coeficiente de determinação dos efeitos de parcela $(\%)$ & 6,14 & 8,29 & 2,42 & 1,39 \\
\hline Coeficiente de variação genética aditiva individual (\%) & 7,45 & 8,10 & 6,50 & 6,26 \\
\hline Coeficiente de variação experimental (\%) & 15,47 & 6,58 & 7,18 & 2,28 \\
\hline Coeficiente de variação relativa (CVgi/CVe) & 0,48 & 1,23 & 0,90 & 2,75 \\
\hline Média geral & 6,1121 & 0,3177 & 5,2033 & 98,4856 \\
\hline Média das populações $\mathrm{F}_{3}$ & 5,7205 & 0,3005 & 4,9931 & 99,4448 \\
\hline
\end{tabular}


concomitantemente os maiores valores genéticos quanto à produtividade de grãos, ao índice de colheita e à altura de planta; e sete $(3,4,5,15,16,18$ e 21) tiveram os maiores valores quanto ao índice de colheita e à altura de planta (Tabela 3). A população 21 figurou, ao mesmo tempo, entre as dez superiores nos quatro caracteres analisados. Essas observações evidenciam a possibilidade de encontrar, dentro dessas populações, indivíduos que concentram alelos favoráveis para os quatro caracteres.
Os ganhos preditos com a seleção de indivíduos superiores, com base no efeito aditivo, foram expressivos. Considerando-se $10 \%$ de seleção, que corresponde a 836 indivíduos selecionados quanto à produtividade de grãos e ao índice de colheita, e de 850 indivíduos quanto ao número de perfilhos e à altura de planta, os ganhos foram de $7,3 \%$ para a produtividade de grãos, $12,5 \%$ para o índice de colheita e 9,1\% para o número de perfilhos e a altura de planta (Tabela 4). Os ganhos por seleção quanto ao índice de colheita,

Tabela 3. Efeito genético aditivo (a), valor genético aditivo ( $\mathrm{u}+\mathrm{a})$ e ganho genético acumulado (GA), preditos para 36 populações $(\mathrm{POP})$ segregantes $\mathrm{F}_{3}$ de trigo, quanto aos caracteres produtividade de grãos, índice de colheita, número de perfilhos e altura de plantas ${ }^{(1)}$

\begin{tabular}{|c|c|c|c|c|c|c|c|c|c|c|c|c|c|c|c|c|}
\hline \multirow[t]{2}{*}{ Ordem } & \multicolumn{4}{|c|}{ Produtividade de grãos } & \multicolumn{4}{|c|}{ Índice de colheita } & \multicolumn{4}{|c|}{ Número de perfilhos } & \multicolumn{4}{|c|}{ Altura de planta } \\
\hline & POP & $\mathrm{a}$ & $\mathrm{u}+\mathrm{a}$ & GA (\%) & POP & $\mathrm{a}$ & $\mathrm{u}+\mathrm{a}$ & GA ( $(\%)$ & POP & $\mathrm{a}$ & $\mathrm{u}+\mathrm{a}$ & GA ( $(\%)$ & POP & $\mathrm{a}$ & $\mathrm{u}+\mathrm{a}$ & GA $(\%)$ \\
\hline 1 & 19 & 0,4465 & 6,56 & 7,8 & 19 & 0,0425 & 0,36 & 14,1 & 30 & 0,5402 & 5,74 & 10,8 & 17 & $-10,62$ & 87,86 & 10,68 \\
\hline 2 & 21 & 0,4396 & 6,55 & 7,7 & 5 & 0,0363 & 0,35 & 13,1 & 36 & 0,4210 & 5,62 & 9,6 & 5 & $-8,64$ & 89,85 & 9,68 \\
\hline 3 & 3 & 0,3708 & 6,48 & 7,3 & 15 & 0,0317 & 0,35 & 12,3 & 32 & 0,4064 & 5,61 & 9,1 & 15 & $-8,58$ & 89,90 & 9,33 \\
\hline 4 & 1 & 0,3471 & 6,46 & 7,0 & 3 & 0,0306 & 0,35 & 11,7 & 31 & 0,4036 & 5,61 & 8,9 & 23 & $-7,64$ & 90,85 & 8,92 \\
\hline 5 & 14 & 0,3367 & 6,45 & 6,8 & 6 & 0,0278 & 0,35 & 11,2 & 21 & 0,3569 & 5,56 & 8,5 & 3 & $-7,01$ & 91,47 & 8,55 \\
\hline 6 & 15 & 0,3216 & 6,43 & 6,6 & 16 & 0,0275 & 0,35 & 10,9 & 27 & 0,3190 & 5,52 & 8,2 & 16 & $-6,18$ & 92,31 & 8,16 \\
\hline 7 & 18 & 0,3027 & 6,41 & 6,4 & 18 & 0,0264 & 0,34 & 10,6 & 24 & 0,2537 & 5,46 & 7,7 & 22 & $-5,7$ & 92,79 & 7,81 \\
\hline 8 & 13 & 0,2814 & 6,39 & 6,2 & 4 & 0,0247 & 0,34 & 10,3 & 8 & 0,2220 & 5,43 & 7,3 & 21 & $-5,07$ & 93,42 & 7,47 \\
\hline 9 & 5 & 0,2576 & 6,37 & 6,0 & 1 & 0,0219 & 0,34 & 10,0 & 26 & 0,2190 & 5,42 & 7,0 & 4 & $-4,66$ & 93,83 & 7,16 \\
\hline 10 & 2 & 0,2089 & 6,32 & 5,8 & 21 & 0,0214 & 0,34 & 9,7 & 33 & 0,1664 & 5,37 & 6,6 & 18 & $-4,24$ & 94,25 & 6,87 \\
\hline 11 & 16 & 0,1979 & 6,31 & 5,6 & 22 & 0,0205 & 0,34 & 9,4 & 19 & 0,1300 & 5,33 & 6,3 & 12 & $-3,63$ & 94,85 & 6,58 \\
\hline 12 & 6 & 0,1720 & 6,28 & 5,4 & 17 & 0,0168 & 0,33 & 9,1 & 18 & 0,0973 & 5,30 & 5,9 & 29 & $-3,45$ & 95,04 & 6,32 \\
\hline 13 & 30 & 0,1516 & 6,26 & 5,2 & 13 & 0,0099 & 0,33 & 8,7 & 9 & 0,0789 & 5,28 & 5,6 & 6 & $-3,16$ & 95,33 & 6,08 \\
\hline 14 & 4 & 0,1491 & 6,26 & 5,0 & 24 & 0,0093 & 0,33 & 8,3 & 12 & 0,0544 & 5,26 & 5,2 & 33 & $-2,96$ & 95,53 & 5,86 \\
\hline 15 & 31 & 0,1379 & 6,25 & 4,8 & 14 & 0,0048 & 0,32 & 7,8 & 25 & 0,0486 & 5,25 & 5,0 & 19 & $-2,31$ & 96,18 & 5,62 \\
\hline 16 & 22 & 0,1239 & 6,24 & 4,6 & 12 & 0,0027 & 0,32 & 7,4 & 29 & 0,0421 & 5,25 & 4,7 & 27 & $-2,14$ & 96,34 & 5,40 \\
\hline 17 & 24 & 0,0645 & 6,18 & 4,4 & 2 & $-0,0028$ & 0,31 & 6,9 & 1 & 0,0397 & 5,24 & 4,5 & 9 & $-1,85$ & 96,64 & 5,20 \\
\hline 18 & 17 & 0,0565 & 6,17 & 4,2 & 33 & $-0,0039$ & 0,31 & 6,4 & 2 & 0,0328 & 5,24 & 4,3 & 24 & $-1,77$ & 96,71 & 5,01 \\
\hline 19 & 12 & 0,0203 & 6,13 & 4,0 & 23 & $-0,0044$ & 0,31 & 6,0 & 3 & 0,0242 & 5,23 & 4,1 & 35 & $-1,09$ & 97,40 & 4,80 \\
\hline 20 & 36 & $-0,0076$ & 6,10 & 3,8 & 26 & $-0,0050$ & 0,31 & 5,6 & 7 & 0,0155 & 5,22 & 3,9 & 13 & 0,18 & 98,67 & 4,55 \\
\hline 21 & 34 & $-0,0092$ & 6,10 & 3,6 & 31 & $-0,0073$ & 0,31 & 5,3 & 35 & $-0,0054$ & 5,20 & 3,7 & 11 & 1,78 & 100,27 & 4,25 \\
\hline 22 & 33 & $-0,0916$ & 6,02 & 3,4 & 36 & $-0,009$ & 0,31 & 4,9 & 15 & $-0,0108$ & 5,19 & 3,5 & 34 & 2,07 & 100,56 & 3,96 \\
\hline 23 & 32 & $-0,0965$ & 6,02 & 3,2 & 25 & $-0,0116$ & 0,31 & 4,5 & 20 & $-0,0590$ & 5,14 & 3,3 & 14 & 2,11 & 100,59 & 3,70 \\
\hline 24 & 26 & $-0,1013$ & 6,01 & 3,0 & 32 & $-0,0120$ & 0,31 & 4,1 & 13 & $-0,0716$ & 5,13 & 3,1 & 26 & 2,67 & 101,16 & 3,43 \\
\hline 25 & 10 & $-0,1379$ & 5,97 & 2,8 & 7 & $-0,0121$ & 0,31 & 3,8 & 34 & $-0,0826$ & 5,12 & 2,9 & 1 & 2,73 & 101,22 & 3,18 \\
\hline 26 & 9 & $-0,2060$ & 5,91 & 2,5 & 34 & $-0,0128$ & 0,30 & 3,5 & 28 & $-0,1324$ & 5,07 & 2,7 & 32 & 3,56 & 102,04 & 2,92 \\
\hline 27 & 27 & $-0,2183$ & 5,89 & 2,3 & 9 & $-0,0144$ & 0,30 & 3,2 & 6 & $-0,1400$ & 5,06 & 2,5 & 31 & 3,78 & 102,26 & 2,67 \\
\hline 28 & 25 & $-0,2568$ & 5,86 & 2,0 & 10 & $-0,0214$ & 0,30 & 2,8 & 14 & $-0,1420$ & 5,06 & 2,3 & 7 & 4,86 & 103,35 & 2,40 \\
\hline 29 & 28 & $-0,2708$ & 5,84 & 1,8 & 30 & $-0,0217$ & 0,30 & 2,5 & 10 & $-0,1554$ & 5,05 & 2,1 & 10 & 5,33 & 103,82 & 2,14 \\
\hline 30 & 35 & $-0,3015$ & 5,81 & 1,6 & 28 & $-0,0221$ & 0,30 & 2,2 & 22 & $-0,2243$ & 4,98 & 1,9 & 28 & 6,33 & 104,81 & 1,85 \\
\hline 31 & 7 & $-0,3289$ & 5,78 & 1,3 & 27 & $-0,0238$ & 0,29 & 1,8 & 5 & $-0,3374$ & 4,87 & 1,6 & 20 & 6,54 & 105,02 & 1,58 \\
\hline 32 & 29 & $-0,3365$ & 5,78 & 1,1 & 20 & $-0,0266$ & 0,29 & 1,5 & 17 & $-0,4192$ & 4,78 & 1,3 & 2 & 6,96 & 105,45 & 1,31 \\
\hline 33 & 20 & $-0,4539$ & 5,66 & 0,8 & 35 & $-0,0279$ & 0,29 & 1,2 & 16 & $-0,5095$ & 4,69 & 1,0 & 36 & 7,36 & 105,85 & 1,05 \\
\hline 34 & 23 & $-0,4857$ & 5,63 & 0,6 & 29 & $-0,0306$ & 0,29 & 0,8 & 23 & $-0,5108$ & 4,69 & 0,6 & 30 & 8,31 & 106,80 & 0,77 \\
\hline 35 & 8 & $-0,5213$ & 5,59 & 0,3 & 11 & $-0,0346$ & 0,28 & 0,5 & 4 & $-0,5223$ & 4,68 & 0,3 & 25 & 12,17 & 110,65 & 0,40 \\
\hline 36 & 11 & $-0,5631$ & 5,55 & 0,0 & 8 & $-0,0506$ & 0,27 & 0,0 & 11 & $-0,5491$ & 4,65 & 0,0 & 8 & 13,93 & 112,42 & 0,00 \\
\hline
\end{tabular}

${ }^{(1)}$ Valor genético obtido com a média geral e o ganho acumulado predito com base na média das populações. 
Tabela 4. Efeito genético aditivo (a), valor genético aditivo individual $(\mathrm{u}+\mathrm{a})$ e ganho genético acumulado (GA, \%), preditos com seleção de 10 e 20\% dos melhores indivíduos (Planta), quanto aos caracteres produção de grãos, índice de colheita, número de perfilhos e altura de plantas de trigo ${ }^{(1)}$.

\begin{tabular}{|c|c|c|c|c|c|c|c|c|c|c|c|c|c|c|c|c|c|c|c|c|}
\hline \multirow[t]{2}{*}{ Ordem } & \multicolumn{5}{|c|}{ Produtividade de grãos } & \multicolumn{5}{|c|}{ Índice de colheita } & \multicolumn{5}{|c|}{ Número de perfilhos } & \multicolumn{5}{|c|}{ Altura de planta } \\
\hline & POP & Planta & $\mathrm{a}$ & $\mathrm{u}+\mathrm{a}$ & $\overline{\mathrm{GA}}$ & POP & Planta & $\mathrm{a}$ & $\mathrm{u}+\mathrm{a}$ & $\overline{\mathrm{GA}}$ & POP & Planta & $\mathrm{a}$ & $\mathrm{u}+\mathrm{a}$ & GA & POP & Planta & $\mathrm{a}$ & $\mathrm{u}+\mathrm{a}$ & GA \\
\hline 1 & 19 & 31 & 0,851 & 963 & 14,9 & 19 & 31 & 0,077 & 0,395 & 25,6 & 30 & 40 & 62 & 5,865 & 13,3 & 17 & 21 & $-13,788$ & 4,698 & 13,9 \\
\hline 2 & 21 & & 0,631 & 743 & 3,0 & 19 & 82 & 0,077 & 0,395 & 5,6 & 30 & 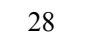 & 0,647 & 5,850 & 13,1 & 17 & & 21 & 4,864 & 13,8 \\
\hline 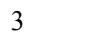 & 19 & 86 & 0,625 & 6,737 & 12,3 & 19 & 63 & 0,064 & 0,382 & 24,1 & 30 & 8 & 0,620 & 5,823 & 12,9 & 17 & 15 & $-13,560$ & 84,925 & 13,7 \\
\hline & 21 & 7 & 0,568 & 6,680 & 11,7 & 19 & 66 & 0,055 & 0,373 & 22,7 & 30 & 22 & 0,620 & 5,823 & 12,8 & 17 & 16 & $-13,249$ & 85,237 & 13,6 \\
\hline & 19 & 4 & 0,565 & 6,677 & 11,3 & 19 & 72 & 0,053 & 0,371 & 21,7 & 30 & 30 & 0,620 & 5,823 & 12,7 & 17 & 63 & $-13,145$ & 85,341 & 13,5 \\
\hline & 19 & 2 & 0,556 & 6,668 & 11,1 & 19 & 81 & 0,052 & 0,370 & 21,0 & 30 & 53 & 0,620 & 5,823 & 12,6 & 17 & 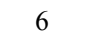 & $-12,729$ & 85,757 & 13,4 \\
\hline & 2 & 1 & 0,554 & 6,666 & 10,9 & 19 & 6 & 0,052 & 0,370 & 20,5 & 30 & 68 & 0,620 & 5,823 & 12,6 & 17 & 31 & $-12,521$ & 85,964 & 13,3 \\
\hline 8 & 21 & 20 & 0,541 & 6,653 & 10,7 & 19 & 37 & 0,051 & 0,369 & 20,0 & 30 & 50 & 0,605 & 5,808 & 12,5 & 17 & 19 & $-12,521$ & 85,964 & 13,2 \\
\hline & 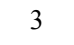 & 4 & 0,534 & 6,646 & 10,5 & 19 & 4 & 0,051 & 0,369 & 19,7 & 30 & 5 & 0,605 & 5,808 & 12,5 & 17 & 1 & -12 , & 85,964 & 13,1 \\
\hline 10 & 21 & 16 & 0,532 & 6,644 & 10,4 & 19 & 31 & 0,051 & 0,369 & 19,4 & 30 & 12 & 0,603 & 5,807 & 12,5 & 17 & 60 & $-12,479$ & 86,007 & 13,1 \\
\hline 1 & 21 & 4 & 0,530 & 6,642 & 10,3 & 16 & 8 & 0,051 & 0,369 & 19,2 & 30 & 4 & 0,603 & 5,807 & 12,4 & 17 & 65 & $-12,417$ & 86,068 & 13,0 \\
\hline 12 & 1 & 31 & 0,526 & 6,638 & 10,2 & 19 & 36 & 0,050 & 0,368 & 19,0 & 30 & 43 & 0,603 & 5,807 & 12,4 & 17 & 76 & $-12,375$ & 86,111 & 13,0 \\
\hline 3 & 19 & 8 & 0,524 & 6,636 & 10,1 & 19 & 19 & 0,049 & 0,367 & 18,8 & 30 & 61 & 0,603 & 5,807 & 12,4 & 17 & 64 & $-12,333$ & 86,152 & 12,9 \\
\hline $0 \%$ & 15 & 54 & 0,351 & 6,463 & 7,3 & 15 & 42 & 0,031 & 0,349 & 12,5 & 36 & 62 & 0,384 & 5,588 & 9,1 & 16 & 35 & $-7,495$ & 90,990 & 9,1 \\
\hline $20 \%$ & 13 & 38 & 0,286 & 6,398 & 6,4 & 1 & 87 & 0,025 & 0,343 & 10,9 & 24 & 14 & 0,236 & 5,439 & 7,7 & 3 & 21 & $-5,411$ & 93,075 & 7,8 \\
\hline \multicolumn{3}{|c|}{ Média geral } & & 6,112 & & & & & 0,318 & & & & & 5,203 & & & & & 98,486 & \\
\hline \multicolumn{4}{|c|}{ Média das populações } & 5,720 & & & & & 0,300 & & & & & 4,993 & & & & & 99,445 & \\
\hline
\end{tabular}

${ }^{(1)}$ Valor genético obtido com a média geral e o ganho acumulado, preditos com base na média das populações. ${ }^{(2)}$ As pressões de seleção de 10 e $20 \%$ correspondem aos valores de 836 e 1.672 indivíduos, quanto à produtividade de grãos e ao índice de colheita, e de 850 e 1.699 indivíduos, quanto ao número de perfilhos e à altura de planta, respectivamente. POP, população de origem.

em trigo, são normalmente muito baixos. Isso realça ainda mais os $12,5 \%$ obtidos no presente trabalho. Com a percentagem de seleção de $20 \%$, que implica 1.672 indivíduos selecionados quanto à produtividade de grãos e ao índice de colheita, e de 1.699 indivíduos quanto ao número de perfilhos e à altura de planta, os ganhos variaram entre $6,4 \%$ de produtividade e $10,9 \%$ de índice de colheita. Esses ganhos foram preditos a partir do valor genético médio das populações e do valor genético médio dos indivíduos selecionados; este último representa a soma do efeito aditivo médio dos indivíduos selecionados e o valor genético médio das populações.

\section{Conclusões}

1. A variação ambiental dentro de parcela ou entre plantas apresenta contribuição majoritária para a variância fenotípica total, na avaliação de plantas individuais de trigo.

2. A herdabilidade individual contribui para maior ganho genético, nos caracteres altura de planta e índice de colheita, com o uso do BLUP individual.

3. As populações Embrapa22/BRS207, Embrapa22/ VI98053, Embrapa22/IVI01041, BRS254/BRS207,
BRS254/VI98053, BRS254/UFVT1Pioneiro e BRS264/BRS207 destacam-se por apresentar valor genético aditivo elevado em dois ou mais caracteres.

\section{Agradecimentos}

Ao Conselho Nacional de Desenvolvimento Científico e Tecnológico $(\mathrm{CNPq})$ e à Coordenação de Aperfeiçoamento de Pessoal de Nível Superior (Capes), pelo apoio financeiro.

\section{Referências}

BARROS, F.R.; ANUNCIAÇÃO FILHO, C.J. da; ROCHA, M. de M.; NUNES, J.A.R.; SILVA, K.J.D. e; FREIRE FILHO, F.R.; RIBEIRO, V.Q. Potencial genético de progênies de feijão-caupi segregantes quanto ao tipo da inflorescência. Pesquisa Agropecuária Brasileira, v.46, p.182-189, 2011. DOI: 10.1590/ S0100-204X2011000200010.

BERTOLDO, J.G.; COIMBRA, J.L.M.; GUIDOLIN, A.F.; NODARI, R.O.; ELIAS, H.T.; BARILI, L.D.; VALE, N.M. do; ROZZETTO, D.S. Rendimento de grãos em feijão preto: o componente que mais interfere no valor fenotípico é o ambiente. Ciência Rural, v.39, p.1974-1982, 2009. DOI: 10.1590/ S0103-84782009005000166.

BORGES, V.; SOARES, A.A.; REIS, M.S.; RESENDE, M.D.V.; CORNÉLIO, V.M.O.; LEITE, N.A.; VIEIRA, A.R. Desempenho 
genotípico de linhagens de arroz de terras altas utilizando metodologia de modelos mistos. Bragantia, v.69, p.833-841, 2010. DOI: $10.1590 /$ S0006-87052010000400008.

CARGNIN, A.; SOUZA, M.A. de; FRONZA, V. Progress in breeding of irrigated wheat for the Cerrado region of Brazil. Crop Breeding and Applied Biotechnology, v.8, p.39-46, 2008. DOI: 10.12702/1984-7033.v08n01a06.

CARGNIN, A.; SOUZA, M.A. de; FRONZA, V.; FOGAÇA, C.M. Genetic and environmental contributions to increased wheat yield in Minas Gerais, Brazil. Scientia Agricola, v.66, p.317-322, 2009. DOI: $10.1590 / \mathrm{S} 0103-90162009000300006$.

CHIORATO, A.F.; CARBONELL, S.A.M.; DIAS, L.A. dos S.; RESENDE, M.D.V. Prediction of genotypic values and estimation of genetic parameters in common bean. Brazilian Archives of Biology and Technology, v.51, p.465-472, 2008. DOI: 10.1590/ S1516-89132008000300005.

COIMBRA, J.L.M.; KOPP, M.M.; SOUZA, V.Q. de; BENIN, G.; MARCHIORO, V.S.; CARVALHO, F.I.F. de; OLIVEIRA, A.C. de. Prediction of genetic value in $\mathrm{F}_{3}$ populations of Avena sativa $\mathrm{L}$. using REML/BLUP. Crop Breeding and Applied Biotechnology, v.5, p.265-271, 2005. DOI: 10.12702/1984-7033.v05n03a02.

DUARTE, J.B.; VENCOVSKY, R. Estimação e predição por modelo linear misto com ênfase na ordenação de médias de tratamentos genéticos. Scientia Agricola, v.58, p.109-117, 2001. DOI: 10.1590/S0103-90162001000100017.

FREITAS, I.L. de J.; AMARAL JÚNIOR, A.T. do; VIANA, A.P.; PENA, G.F.; CABRAL, P. da S.; VITTORAZZI, C.; SILVA, T.R. da C. Ganho genético avaliado com índices de seleção e com REML/Blup em milho-pipoca. Pesquisa Agropecuária Brasileira, v.48, p.1464-1471, 2013. DOI: 10.1590/S0100-204X2013001100007.

MAIA, M.C.C.; RESENDE, M.D.V. de; OLIVEIRA, L.C. de; ÁlVARES, V. de S.; MACIEL, V.T.; LIMA, A.C. de. Seleção de clones experimentais de cupuaçu para características agroindustriais via modelos mistos. Revista Agro@mbiente On-line, v.5, p.35-43, 2011.

MENDES, M.P.; RAMALHO, M.A.P.; ABREU, Â. de F.B. Strategies for selecting individuals in common bean breeding programs. Bean Improvement Cooperative, v.54, p.68-69, 2011.

NEDEL, J.L. Progresso genético no rendimento de grãos de cultivares de trigo lançadas para cultivo entre 1940 e 1992. Pesquisa Agropecuária Brasileira, v.29, p.1565-1570, 1994.

NUNES, J.A.R.; RAMALHO, M.A.P.; FERREIRA, D.F. Inclusion of genetic relationship information in the pedigree selection method using mixed models. Genetics and Molecular Biology, v.31, p.73-78, 2008. DOI: 10.1590/ S1415-47572008000100015.
OAKEY, H.; VERBYLA, A.; PITCHFORD, W.; CULLIS, B.; KUCHEL, H. Joint modelling of additive and non-additive genetic line effects in single field trials. Theoretical and Applied Genetics, v.113, p.809-819, 2006. DOI: 10.1007/s00122-006-0333-z.

PINHEIRO, L.C. de M.; GOD, P.I.V.G.; FARIA, V.R.; OLIVEIRA, A.G.; HASUI, A.A.; PINTO, E.H.G.; ARRUDA, K.M.A.; PIOVESAN, N.D.; MOREIRA, M.A. Parentesco na seleção para produtividade e teores de óleo e proteína de soja via modelos mistos. Pesquisa Agropecuária Brasileira, v.48, p.1246-1253, 2013. DOI: 10.1590/S0100-204X2013000900008.

RAMALHO, M.A.P.;ARAÚJO, L.C. de A. Breeding self-pollinated plants. Crop Breeding and Applied Biotechnology, v.11, p.1-7, 2011. DOI: 10.1590/S1984-70332011000500002.

RAMALHO, M.A.P.; CARVALHO, B.L.; NUNES, J.A.R. Perspectives for the use of quantitative genetics in breeding of autogamous plants. ISRN Genetics, v.2013, article ID 718127, 2013. DOI: $10.5402 / 2013 / 718127$

RESENDE, M.D.V. de. Análise estatística de modelos mistos via REML/BLUP na experimentação em melhoramento de plantas perenes. Colombo: Embrapa Florestas, 2000. 101p. (Embrapa Florestas. Documentos, 47).

RESENDE, M.D.V. de. Genética biométrica e estatística no melhoramento de plantas perenes. Brasília: Embrapa Informação Tecnológica; Colombo: Embrapa Florestas, 2002. 975p.

RESENDE, M.D.V. de. Matemática e estatística na análise de experimentos e no melhoramento genético. Colombo: Embrapa Florestas, 2007a. 561p.

RESENDE, M.D.V. de. Métodos estatísticos ótimos na análise de experimentos de campo. Colombo: Embrapa Florestas, 2004. 57 p. (Embrapa Florestas. Documentos, 100).

RESENDE, M.D.V. de. Software SELEGEN-REML/BLUP: sistema estatístico e seleção genética computadorizada via modelos lineares mistos. Colombo: Embrapa Florestas, 2007b. 359p.

RESENDE, M.D.V. de; DUARTE, J.B. Precisão e controle de qualidade em experimentos de avaliação de cultivares. Pesquisa Agropecuária Tropical, v.37, p.182-194, 2007.

RESENDE, M.D.V. de; HIGA, A.R. Estimação de valores genéticos no melhoramento de Eucalyptus: seleção em um caráter com base em informações do individuo e de seus parentes. Boletim de Pesquisa Florestal, n.28-29, p.11-36, 1994.

REUNIÃO DA COMISSÃO BRASILEIRA DE PESQUISA DE TRIGO E TRITICALE, 1., 2007, Londrina. Informações técnicas para a safra 2008: trigo e triticale. Londrina: Embrapa Soja, 2008. 147 p. (Embrapa Soja. Documentos, 301).

VENCOVSKY, R.; BARRIGA, P. Genética biométrica no fitomelhoramento. Ribeirão Preto: Sociedade Brasileira de Genética, 1992. 496p.

Recebido em 13 de maio de 2013 e aprovado em 9 de outubro de 2014

Pesq. agropec. bras., Brasília, v.49, n.11, p.882-890, nov. 2014 DOI: $10.1590 / \mathrm{S} 0100-204 X 2014001100007$ 